\title{
The growth mechanism of ordered nanoporous alumina
}

\author{
Marat Makhmud-Akhunov ${ }^{1, *}$, Artem Adamovich $^{1}$, and Igor Yavtushenko ${ }^{1}$ \\ ${ }^{1}$ Ulyanovsk State University, Ulyanovsk, 432017 Leo Tolstoy Street, 42, Russia
}

\begin{abstract}
The paper scrutinizes the peculiarities of porous alumina growth during electrochemical anodic oxidation in the orthophosphoric acid solution at constant potential of 100 Volt. On the basis of atomic-force microscopy data the paper presents consecutive stages of ordering pores in the oxide with the formation of hexagonal dense package after two-stage anodic treatment.
\end{abstract}

\section{Introduction}

Oxide films on the surface of metals and semiconductors are widely used as electric isolation, corrosion-inhibiting covering, and intermediate buffer layers during hermetic metal brazing with different glasses, etc. Dielectric films bear the functional load in semiconductor condensers, transistors of the type metal-oxide-semiconductor (MOS), and etc.

Nowadays much attention is given to nanoporous oxides [1, 2], which are anodestructured in oxygen-containing electrolytes. Such structures often have nonstandard physical, optical and electric properties, including high photosensitivity [3] and catalytic selectivity, which gives an opportunity of practical use of nanoporous structures as extremely sensitive sensors of gas environment components [4]. The priority of such structures is noticeable in photocatalysis [5], in production technique of lithium-ion batteries of extended capacity [6], in solar cells of new generation [7]. However, despite practical success in the sphere of getting porous oxide structures, the description of mechanism and pores formation kinetics is not sufficiently presented; most of works are focused on the description of methods and techniques of getting highly-ordered porous structures, on the modification of their properties and on the application area without a detailed description of the ordering mechanism. Within the scope of the research this paper will present consecutive stages of the growth of porous anodic alumina, having an ordered honeycomb structure.

\section{Experiment}

During the research we used polycrystalline Al samples of a cylindrical shape $\left(\mathrm{S}=0.2 \mathrm{~cm}^{2}\right)$, produced from cast especially pure $\mathrm{Al}$ of brand A999. The internal stress

\footnotetext{
* Corresponding author: maratmau@mail.ru
} 
relieving was carried out by two-hour annealing of the researched samples at temperature $\mathrm{T}=450^{\circ} \mathrm{C}$. The samples were poured with epoxy and kept at room temperature during 24 hours. The working surface of each sample consecutively underwent grinding and polishing till the smooth state with further chemical polishing in the solution $\mathrm{H}_{3} \mathrm{PO}_{4}: \mathrm{HNO}_{3}=85: 15$ at $85{ }^{\circ} \mathrm{C}$ during $5 \mathrm{~min}$ [8]. For the neutralization of the acid residue on the surface the sample was kept for $5 \mathrm{~min}$ in $0.1 \mathrm{M}$ solution $\mathrm{NaOH}$ and was washed properly with distilled water, after that the sample was placed in the cell. The structure of the cell is presented in [9].

To implement two-stage anodic treatment the selective removal of the "sacrificial" oxide layer after the first stage of oxidation was done in the mixture of $20 \mathrm{gr} / \mathrm{C} \mathrm{CrO}_{3}$ and 35 $\mathrm{ml} / 1 \mathrm{H}_{3} \mathrm{PO}_{4}$ at $85^{\circ} \mathrm{C}$ during $30 \mathrm{~min}$.

The experiments on porous alumina were carried out under controlled potential conditions at $\mathrm{U}=100 \mathrm{~V}$ with the lower location of the researched electrode under plumbeous cathode. As an electrolyte $0.75 \mathrm{M}$ solution of orthophosphoric acid was used. The kinetics of the phenomena in progress and the parameters of the oxide layers were judged by the data of atomic-force microscopy (Solver P47-PRO).

\section{Results and discussion}

Due to the little resistance of the metal under research, at the moment of turning on the outer potential the rupture of the natural oxide layer happens, and the high current goes through the sample $(\sim 850 \mathrm{~mA})$. To exclude the rupture the increase of the anodic potential was done with the rate of $1 \mathrm{~V} / \mathrm{s}$ till the necessary value.

The supplied voltage $U$, sent to the electrodes, is redistributed unevenly between anode and cathode, and is under prevailing control of anode-polarized Al electrode, screened by the oxide. The presence of surface imperfection cannot help influencing the redistribution of electric field force lines. The main active centers of current localization can be the boundaries of the grains, dislocation and mechanical defects like protrusions and microscratchings, remaining after the mechanical polishing of the sample. However, the used annealed coarse-grained aluminum allows doing atomic-force analysis within the limits of one single-crystal block, excluding the influence of grain boundaries. Preliminary done chemical polishing of the sample due to corrosion of the surface layer weakens the influence of the rest imperfections. However, the presence of some concentration of noncorroded imperfections leads to the uneven distribution of electric field force lines, and consequently, to the uneven growth of the oxide film in the shape of the islands (Figure 1a).

The formation of the islands is going on continuously because, having reached critical thickness on the prevailing imperfections and roughness, the current is redistributed to other areas with less resistance. This raises the resultant resistance of the aluminum electrode and lowers the current going through the system. Growing, the oxide islands interface between each other through a thin barrier layer, forcing the current to become localized in the gap of the joined protrusions. The rise of the electrolyte chemical activity nearby the anode [10] leads to the local dissolution of the oxide layer. Cavities, filled with the electrolyte, and transforming according to the oxide film growth into pores, are formed in the film. At the regular arrangement of growing hemispherical oxide islands they form a hexagonal dense package. Then each island will be surrounded by six interstices (Figure 1-a), at which the current localization takes place, and consequently the pores formation and corrosion of the support occur. During the oxidation process the expansion and deepening of the corroded zone under each island take place. At the moment of six nearest chemical milling zones contact the tangential growth of the oxide stops, and only deepening of the oxide into the support goes on. As a result, the corroded zones under the oxide film get the shape of hexagons and form a dense package (Figure 1-b). The offered model of the anodic 
dissolution of the aluminum support prognosticates the presence of a non-corroded zone in $\mathrm{Al}$ support straight under the oxide island.
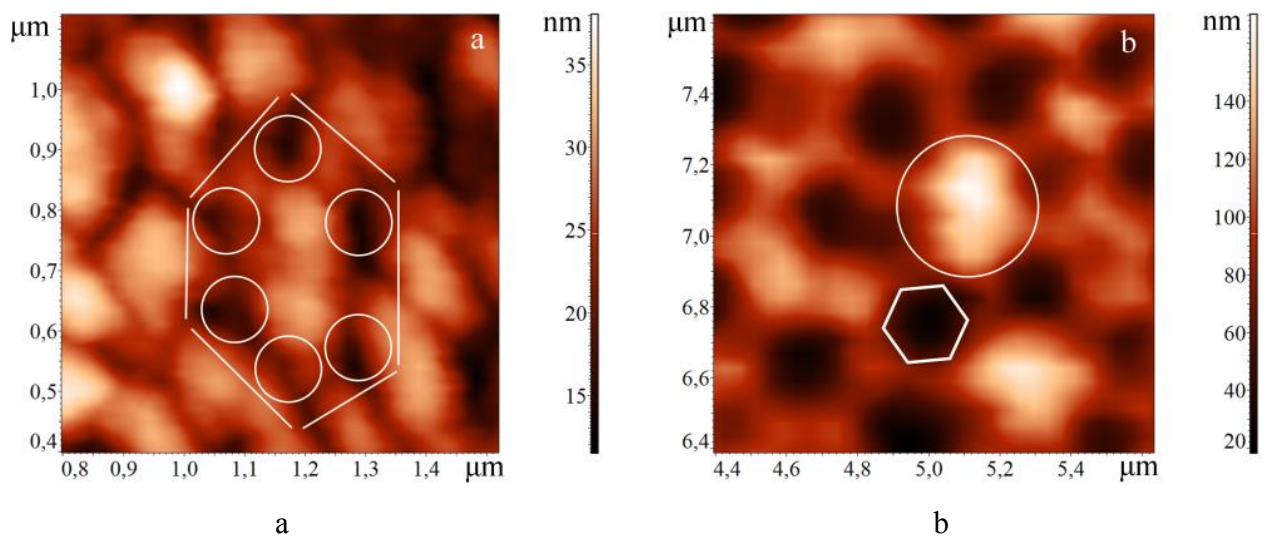

Fig. 1. AFM picture of oxide film surface with the indication of interstices (a) and of $\mathrm{Al}$ support under the film with the indication of the non-corroded zone and of cavities in the shape of hexagons (b) after primary anodic treatment at $\mathrm{U}=100 \mathrm{~V}$ during $30 \mathrm{~min}$.

As it is known, during electrochemical processes, besides the growth of the oxide film, the electrolyte warming up nearby the reaction zone and gas bubbles formation take place. All that violates the regular film growth, and distortions in the regular arrangement of the islands are observed in the oxide. As a result, a typical for this symmetry imperfection of pentagons formation is observed (Figure 2).

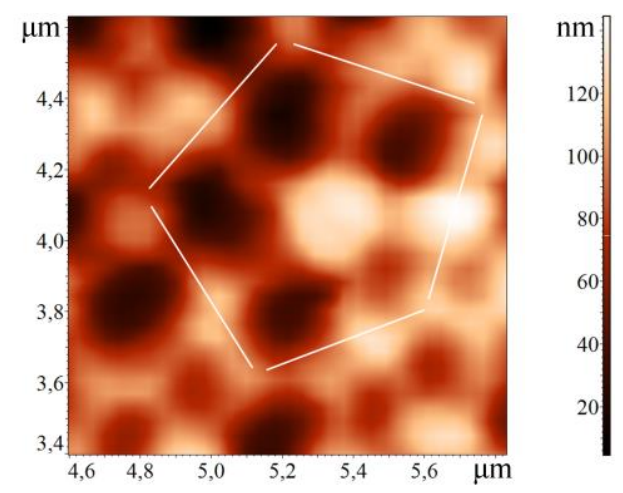

Fig. 2. AFM picture of Al support surface under the oxide film with the breach of the regular arrangement of the corroded areas after the primary anodic treatment at $\mathrm{U}=100 \mathrm{~V}$ during $30 \mathrm{~min}$.

It is significant that the oxide film topology at the second anodic treatment is set by the relief of the corroded Al support surface (Figure 1-b). The current localization mainly occurs on the protrusions, in this case, on the vertices of the hexagons (Figure 1-b), as a result, each pore turns out to be surrounded by six islands (Figure 3-a). Then the noncorroded zone in the support, formed at the primary anodic treatment, (Figure 1-b) is located in the centre of the nearest six oxide islands interface (Figure 3-b). This leads to the current localization exactly in it, i.e. to its corrosion. As a result, after more prolonged oxidation the oxide film surface will be covered with the pores of hexagonal symmetry without imperfection in the package. 

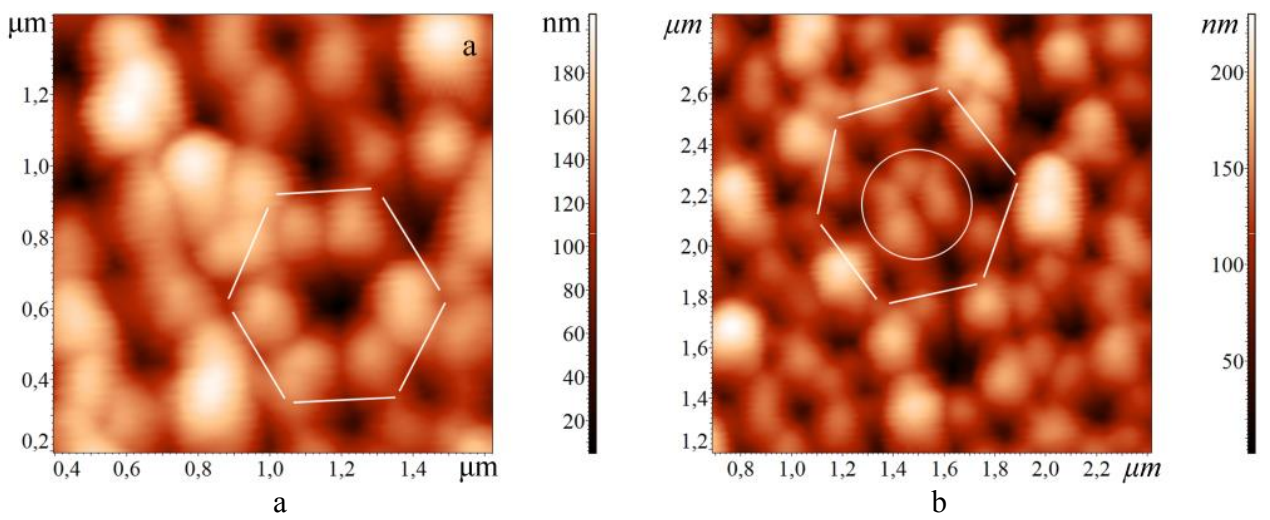

Fig. 3. AFM picture of $\mathrm{Al}$ surface after the second anodic treatment at $\mathrm{U}=100 \mathrm{~V}$ during $90 \mathrm{~min}$. a is the shape of the oxide cell ; $b$ is the dense package with the indication of oxide islands above the noncorroded zone in the support (See Fig. 1-b).

\section{Conclusion}

Thereby, this paper scrutinized the peculiarities of electrochemical formation of porous alumina. Using the method of atomic-force microscopy, we showed the mechanism of pores honeycomb structure formation in the oxide film during two-stage anodic treatment in the self-organized process of the electric field force lines redistribution. The research also showed that the formation of island oxide layer at Stage One of oxidation leads to the incomplete corrosion of $\mathrm{Al}$ support with the presence of untouched areas. The second oxidation of the modified Al surface after the primary treatment sets the relief of the growing oxide layer with the formation of the ordered structure.

This work is supported by the Russian Science Foundation (project no. 19-71-10063).

\section{References}

1. Y. Guo, Li. Zhang, M. Han, X. Wang, J. Xie, L. Deng, R., Soc. Open Sci. 5, 3 (2018)

2. M. Fukuhara, T. Kuroda, F. Hasegawa, Sci. Rep. 6 (2016)

3. A. Hierro-Rodriguez, P. Rocha-Rodrigues, F. Valdés-Bango, et al., J. Phys. D: Appl. Phys. 48, 45 (2015)

4. V. Galstyan, E. Comini, G. Faglia, G. Sberveglieri, Sensors 13, 11 (2013)

5. B. Niu, X. Wang, K. Wu, X. He, R.Zang, Materials 11, 10 (2018)

6. T. Song, H. Han, H. Choi, et al., Nano Res. 7, 4 (2014)

7. T. Balasundaram, K. Raja, Int. J. Chemtech Res. 7, 1 (2014)

8. S. Jin, V. Pham, J. Dickerson, M. Brochu, J. Electrochem. Soc. 162, 11 (2015)

9. A. Orlov, I. Yavtushenko, M. Makhmud-Akhunov, Phys. Solid State 57, 8 (2015)

10. U.R. Dotel, M.O. Sydnes, H.Urkedal, T. Hemmingsen, Math. Models Methods Appl. Sci. 8, 11 (2018) 